\title{
A method to implement data extraction using AND optical gate based on SOA-XGM
}

\author{
Ya-lin GUAN \\ College of Information Engineering \\ Communication University of China \\ Beijing, China
}

\author{
Rui-dong WANG \\ College of Information Engineering \\ Communication University of China \\ Beijing, China
}

\author{
Lei ZHANG \\ College of Information Engineering \\ Communication University of China \\ Beijing, China
}

\begin{abstract}
A method was proposed to implement data extraction using AND optical gate based on cross gain modulation (XGM) of semiconductor optical amplifier (SOA). The optical data extraction in DPSK/ASK orthogonal modulation system was achieved with the proposed AND gates, in order to prove its feasibility. Through the simulation, the data extraction in orthogonal modulation system with a 10Gbit/s NRZ pseudo-random payload signal and 2.5Gbit/s label signal was demonstrated.
\end{abstract}

Keywords-optical label; optical logic gate; orthogonal modulation; semiconductor optical amplifier

\section{INTRODUCTION}

Optical swapping and optical computing are the core unit to achieve all optical signals processing in future all optical networks, they all based on optical logic gates, which are core devices to implement optical swapping system and the key factor of decision network performance. All optical logic gates(XOR、AND)are indispensable part for all optical signal processing, which can be used in all-optical clock recovery, all-optical demultiplexing, all-optical data extraction, etc. Therefore, it has great application potential in future all optical high speed communication network and new generation optical computing.

At present, the research of all optical logic gates are focused on the using of nonlinear effect of optical fiber and semiconductor optical amplifier. The nonlinear effect of optical fiber is weak, so that the logic gates based on nonlinear effect of optical fiber will achieve idea effect only by high power input signal or long fiber. SOA become the main functional device to implement all kinds of optical logic gates, because it has many advantages such as small in size, easy to integrate, short response time and strong nonlinear effect. Taking the nonlinear effect advantage of SOA can realize many kinds of optical logic gates, but the design scheme to realize AND gate is relatively less, for example, using two-level SOA's cross gain modulation effect implementing AND gate, using Mach-Zehnder interferometer based on SOA implementing AND gate, etc. optical data extraction is an important part in optical label swapping system, it is the key factor for all optical network. At present, there have been many design and experiment scheme about optical data extraction. These schemes have it own advantages, but they also have some disadvantages. Article [1] realized all optical data extraction by using start bit in data packet generating address. This method has the advantage of easy to realize and doesn't need complicated clock recovery module; but the realization of control pulse division module is closely related to the length of the packet address, it is needed to increase or decrease the same number of delay fiber when the length of address is increased or decreased. Article [2] realize data extraction from packet through the switch module which are consisted of Mach-Zehnder interferometer based on two-level SOA and Fabry-Perot filter, clock recovery module and address-data separation module; this scheme has the advantage of high speed and flexible; but configuration structure is too complex. By contrast, the method to implement data extraction using AND optical gate has the advantage of high speed in data extraction, simple structure and easy to integrate. In this article we put forward a method to implement AND optical gate using two Wideband Traveling Wave SOAs, and in order to prove the AND gate having the ability in information processing, the AND gate is applied to load DPSK/ label ASK orthogonal modulation system to extract data information. Through the simulation, the data extraction in orthogonal modulation system with a $10 \mathrm{Gbit} / \mathrm{s}$ NRZ pseudo-random payload signal and $2.5 \mathrm{Gbit} / \mathrm{s}$ label signal is demonstrated.

\section{OPERATION PRINCIPLE}

The principle to implement AND gate based on cross gain modulation of SOA was shown in Fig.1. The reversal particle number in working substance of SOA would quickly decrease under the action of the strong light if a beam of weak signal light and a beam of strong signal light inject in SOA, gain of SOA would be saturated, the weak signal which was not amplified by SOA would be outputted, this phenomenon was called cross gain modulation effect. pulse signal light which wavelength was $\lambda_{1}$ and probe signal light which wavelength was $\lambda_{2}$ were injected in SOA at the same time, because of the pulse signal intensity fluctuation, the change of carrier number would lead to the change of SOA gain saturation, i.e. the gain of SOA was modulated by the change of pulse signal intensity, it was modulated by the gain of SOA when probe light through the SOA, for signal pulse peak place, the gain of SOA would reach saturation, probe light would be restrained; for signal pulse valley place, the gain of SOA would not reach saturation, probe light would be amplified, as a result, amplitude of probe light would be modulated by the signal light, output probe signal would carry anti-code pulse sequence of the signal light. 
As shown in Fig.1, clock signal and pulse signal B were injected in SOA-1 at the same time, the intensity of the pulse signal B was enough to modulate the gain of SOA-1, and the clock signal passed SOA-1 was modulated by the gain of SOA-1, output of SOA-1 was clock signal which carries the information of pulse sequence $\overline{\mathbf{B}}$. The output pulse signal $\overline{\mathbf{B}}$ of SOA-1 and pulse signal A were injected in SOA-2, the intensity of $\overline{\mathbf{B}}$ was also enough to modulate the gain of SOA-2, the pulse signal A passed SOA-2 was modulated by the gain of SOA-2, output of SOA-2 was pulse signal A which carried the information of pulse signal $\mathrm{B}$, from the final output result, it was equivalent to realize the logic operation $\mathrm{AB}^{[3]}$.

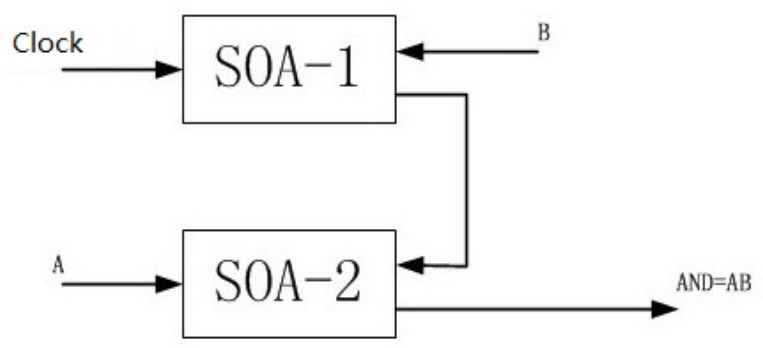

Fig.1 Architecture of AND based on SOA-XGM

The principle to implement optical data extraction using AND gate was depicted in Fig.2. To extract the desired bits from label information, the label information sequence which was needed to define was " 01101 ". By asserting 2 nd and 4th were the data needed extraction from label information, in order to achieve it, 2nd and 4th of control information which decides desired bit position were set as " 1 ", other bits were set as " 0 ", and it meant that pulse sequence of control information was "01010". Through the logical operation of AND gate consisted of SOA-1 and SOA-2, 2nd and 4th of output sequence were the extraction data which we needed.

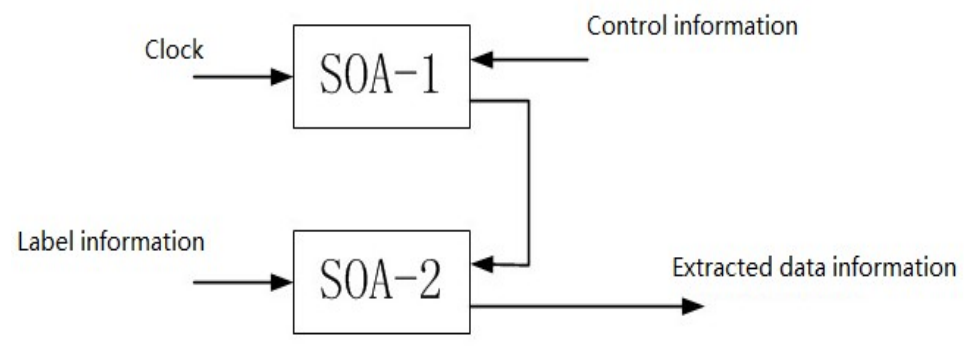

Fig.2 data extraction principle based on AND gate

\section{EXPERIMENT DEMONSTRATION}

The DPSK/ASK orthogonal modulation optical data extraction system structure of simulation was depicted in Fig.3. There were two parts in AND gate optical extraction system. At transmitter, the continuous-wave source was a tunable laser operating at $1550 \mathrm{~nm}$. A pulse generator created a $10 \mathrm{GHz}$ NRZ pseudo-random pulse train, which was synchronously phase-modulated by $10 \mathrm{Gbit} / \mathrm{s}$ payload data through a Mach-Zehnder modulator (MZM) biased at null and driven at $2 \mathrm{~V} \pi$ to achieve phase modulation. Second MZM inserted label information at $2.5 \mathrm{Gbit} / \mathrm{s}$ signal by intensity modulation. The pulse sequence of label was: 01101. The generated DPSK/ASK packet was transmitted $80 \mathrm{~km}$ in standard signal-mode fiber (SSMF) and $16 \mathrm{~km}$ dispersion-compensating fiber (DCF). At data extraction part, using the AND gate composed of two SOAs to extraction optical data, the control information pulse sequence injected in SOA set as " 01010 ". In order to realize the nonlinear effect of SOA easily, the signal were amplified by erbium-doped optical fiber amplifier. In the end, the extracted data was filtered by a Fiber Bragg Grating (FBG) filter and switched by a Photo Detector ${ }^{[4]}$. 


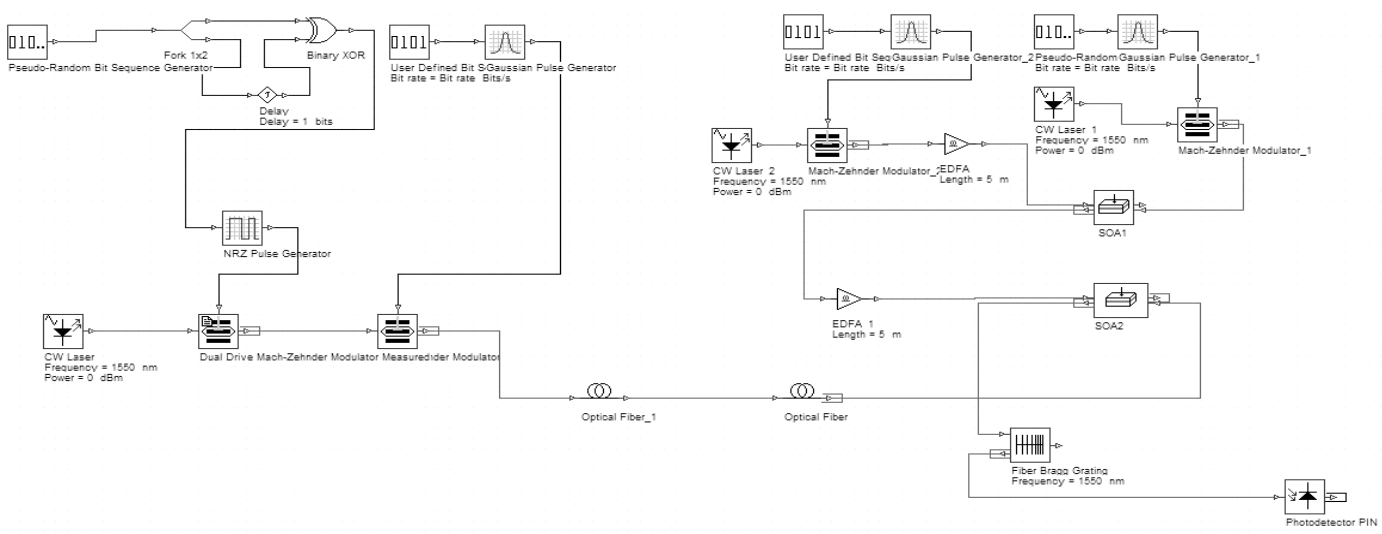

Fig.3 DPSK/ASK orthogonal modulation data extraction system

Time domain waveform of label information after data extraction by AND optical gate based on SOA was shown in Fig.4. The label information was depicted in Fig.4 (a). The extracted data information was depicted in Fig.4 (b). From the extracted data information waveform, pulse information of the second bit was: "1", the fourth bit was " 0 ", consistent with the theoretical value. Therefore, the method to extraction data information using the AND optical gate based on SOA was feasible.

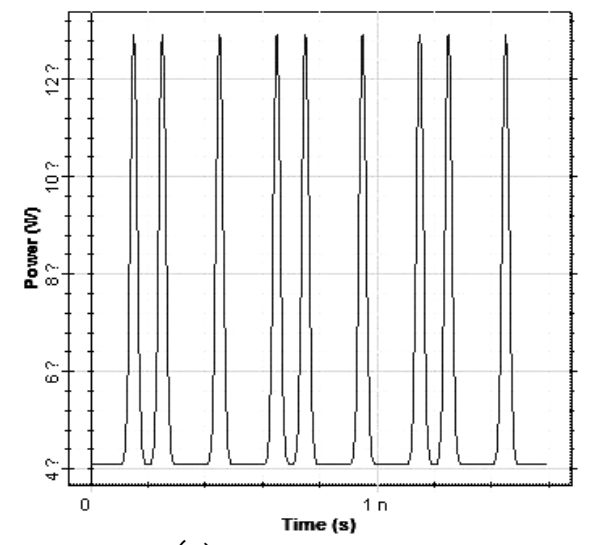

(a) label information

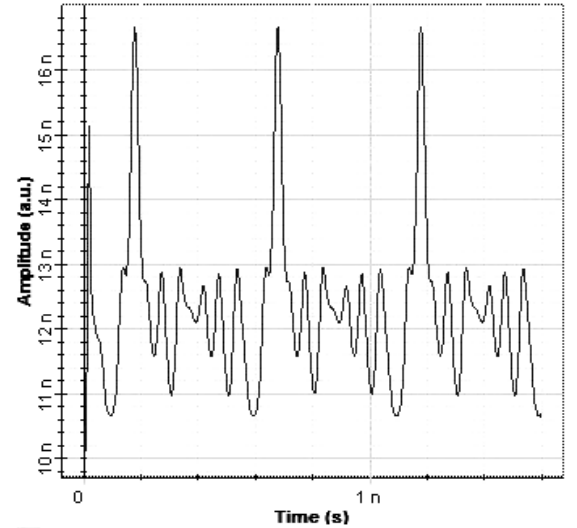

(b) extracted data information

Fig.4 Time domain waveform of label information

\section{CONCLUSION}

By using the SOA cross gain modulation, all optical logic AND gate was realized. The AND gate was applied to load DPSK/ label ASK orthogonal modulation system to extract data information. In the simulation experiment, the label information was extracted. Because of the SOA gain suppression incomplete, the " 0 " output had a pulse fluctuation in the simulation. Further research should be done in order to decrease the pulse fluctuation. Optical data extraction is a way of extraction and recognition optical label in optical domain, and it has extensive application prospect in high speed all-optical networks. The method of this optical data extraction based on optical logic AND gate has high speed of optical data extraction and simple structure. However, the speed of optical data extraction is limited by the carrier recovery time of SOA, this method can only extract optical data lower than $10 \mathrm{Gbit} / \mathrm{s}$.

\section{REFERENCES}

[1] G.L. Papadimitriou. Papazoglou and A.S. Pomportsis, Optical Switching: Switch Fabrics, Techniques, and Architectures, IEEE J. Lightwave Technol, 2003, 21(2): 384-405.

[2] Jourdan, D. Chiaroni, E. Dotaro, et al., The Perspective of Optical Packet Switching in IP-Dominant Backbone and Metropolitan Networks, IEEE Commun. Mag., 2001，39(3):136-141.

[3] Jae Hun Kim, Chang wan son, Yong IL KIM, et al. All-Optical Signal Processing Using Semiconductor Optical Amplifier Based Logic Gates. Numerical Simulation of Semiconductor Optoelectronic Devices, 2003. NUSOD 2003. Proceedings of the IEEE/LEOS 3rd International Conference.

[4] Nan Chi, Jianfeng Zhang, Pablo V. Holm-Nielsen, et al. Transmission and Transparent Wavelength Conversion of an Optically Labeled Signal Using ASK/DPSK Orthogonal Modulation[J].IEEE PHOTONICS TECHNOLOGY LETTERS, VOL. 15, NO. 5, MAY 2003:760-762. 